\title{
Electron beam shaping via laser heater temporal shaping
}

\author{
D. Cesarø, ${ }^{1, *}$ A. Anakru $\odot,{ }^{2}$ S. Carbajo ${ }^{1,3}$ J. Duris, ${ }^{1}$ P. Franz, ${ }^{1}$ S. Li, ${ }^{1}$ \\ N. Sudar $\odot,{ }^{1}$ Z. Zhang $\odot,{ }^{1}$ and A. Marinelli ${ }^{1, \dagger}$ \\ ${ }^{1}$ SLAC National Accelerator Laboratory, 2575 Sand Hill Rd, Menlo Park, California 94025, USA \\ ${ }^{2}$ Cornell University, Ithaca, New York 14850, USA \\ ${ }^{3}$ Electrical and Computer Engineering Department, \\ University of California Los Angeles, California 9004, USA
}

(Received 8 June 2021; accepted 21 October 2021; published 29 November 2021)

\begin{abstract}
Active longitudinal beam optics can help free-electron laser facilities achieve cutting edge performance by optimizing the beam to produce multicolor pulses, suppress caustics, or support attosecond lasing. As the next generation of superconducting accelerators comes online, there is a need to find new elements which can both operate at high-beam power and which offer multiplexing capabilities at $\mathrm{MHz}$ repetition rate. Laser heater shaping promises to satisfy both criteria by imparting a programmable slice-energy spread on a shot-by-shot basis. We use a simple kinetic analysis to show how control of the slice energy spread translates into control of the bunch current profile, and then we present a collection of start-to-end simulations at LCLS-II in order to illustrate the technique.
\end{abstract}

DOI: 10.1103/PhysRevAccelBeams.24.110703

\section{INTRODUCTION}

Facility scale electron accelerators regularly produce high-current, high-brightness electron bunches by accelerating and compressing the bunch from an rf photoinjector. During bunch compression, collective forces and nonlinear optics passively act on the bunch and unavoidably alter the current profile, such that many applications can benefit by actively reshaping the intrabunch current profile. For example, in the context of x-ray free-electron laser facilties (XFELs) [1-7], modulation of the current profile can lead to optimized beam brightness, suppressed horn formation [8], two-color operation [9], and attosecond $\mathrm{x}$-ray pulses [10-13]; while in plasma-wakefield accelerators, shaping of the current profile can lead to maximization of the energy transfer to the witness bunch in high-transformer ratio experiments [14,15].

Existing beam-shaping techniques are often reliant on support from solid target-based collimators [8] and emittance spoilers $[16,17]$. But as the next generation of highaverage power accelerators $[2,18]$ come on-line, these must be replaced by new techniques which are compatible with hundreds of kilowatts of electron beam power. Laser heater shaping is an attractive alternative because it

\footnotetext{
*dcesar@slac.stanford.edu

†marinelli@slac.stanford.edu
}

Published by the American Physical Society under the terms of the Creative Commons Attribution 4.0 International license. Further distribution of this work must maintain attribution to the author(s) and the published article's title, journal citation, and DOI. provides a highly flexible platform for bunch shaping at $\mathrm{MHz}$ repetition rate.

The laser heater uses a wiggler magnet located in the middle of a chicane to couple energy from a laser to an electron beam. The interaction in the wiggler produces a periodic energy modulation which is effectively washed out by the chicane dispersion, such that the net result is a controlled increase in the energy spread of the beam $[19,20]$. Conventionally, this heating is used to suppress the collective instabilities that develop during acceleration and bunch compression (namely the microbunching instability) [21-25]. To accomplish this, the laser duration is much longer than the beam duration such that the heating is applied uniformly along the electron beam. If instead the laser heater intensity profile is modulated as a function of time, then we can create a time-dependent energy spread which can be used to shape the bunch current profile (see Fig. 1).

Such temporal laser shaping was initially proposed to control the temporal properties of XFELs [26] and multicolor generation in a seeded FEL [9]. More recently, a highly periodic laser heater modulation has been used to manipulate the microbunching instability [27]. Laser heater shaping has also been proposed to generate a train of current spikes in order to seed a high-power $\mathrm{THz}$ wiggler [28]. Here, we generalize these methods to the generation of complex current profiles by an arbitrarily shaped laser heater.

High-resolution adaptive temporal shaping of broadband femtosecond laser pulses can be achieved via spectral phase and amplitude shaping or programmable synthesis. For instance, Volume Bragg gratings [29] can be readily used to 


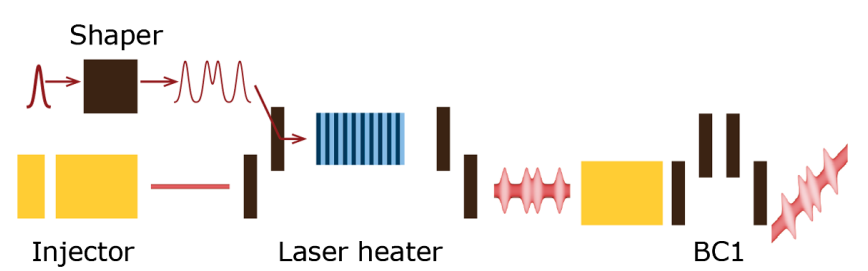

FIG. 1. Cartoon of laser heater shaping at an FEL facility. A high-repetition rate laser can be used to program complicated patterns into the slice energy spread. After bunch compression (BC1), the energy spread is converted to a current modulation.

create single and multiple notches for emittance spoiling or beam slicing, thanks in part to their high-diffraction efficiency, high-damage threshold, and temperature, and optical stability. In the case of more sophisticated or adaptable temporal shapes, spectral amplitude and phase modulators [30,31] can be used, opening the door to machine learning-based control, albeit at damage-limited low-to-moderate peak and average power levels [32] or with a substantially reduced actuation range after amplification of spectrally shaped pulses. Recent demonstrations in the synthesis of $4 \mathrm{D}$ programmable light bullets with adaptable spatiotemporal shapes [33,34] promise to overcome some of these power limitations in the near future.

The article is organized as follows: first we introduce a general theoretical model that describes the evolution of the current profile for an arbitrary laser heater temporal profile going through a single bunch compressor. We then provide a series of numerical case studies in which a temporally shaped laser heater provides useful control of the current profile at an XFEL.

\section{ANALYTICAL MODEL}

Active manipulation of the beam's slice energy spread is an indirect method for shaping its current profile. It works by modulating the beam's longitudinal phase space, such that, immediately following the laser heater, the current is unperturbed, but after bunch compression the energy spread is converted into a current modulation. Even small current modulations can seed further growth downstream via the microbunching instability mechanism. To understand, the extent to which the laser heater can be used to draw structure into the beam's longitudinal phase space, we start with a simple kinetic analysis describing the compression of a beam with slice-dependent energy spread. We then apply the results of this analysis to study a series of applications: the generation of isolated current spikes by a single Gaussian laser heater, a quasiperiodic current profile made by stacking Gaussian pulses, and a highly periodic current profile made from a laser comb.

We start from a reduced model in which we treat the laser heater (the laser, wiggler, and surrounding chicane) as a lumped element which acts on the beam to produce a smooth energy spread. This neglects the possibility of traditional or echo-enabled modulator chicane-based beam-shaping [35], which is justified given the large dispersion and phase space mixing characteristic of laser heater design. Our model also omits any dynamics within the wiggler by assuming that the beam is resonant, that the synchrotron phase advance is small, and that the slippage is negligible. The resulting energy exchange is characterized by a maximum energy change $A(s)$ which is simply proportional to the driving field times the wiggler length:

$$
A(s)=\sqrt{2 Z_{0} I_{l}(s)} \frac{q_{e} K L_{u} J J}{2 \gamma_{0} \gamma m c^{2}},
$$

where $I_{l}(s) \approx 2 P(s) / \pi w_{0}^{2}$ is the onaxis laser intensity (written here in terms of the power, $P_{0}$, and laser waist $\left.w_{0}\right), Z_{0}$ is the impedance of free space, $\gamma m c^{2}$ is the electron energy, $K$ is the undulator parameter, $L_{u}$ is the undulator magnetic length, $\gamma_{0}$ is the reference beam energy, and $J J$ is given by:

$$
J J=J_{0}\left(\frac{K^{2}}{4+2 K^{2}}\right)-J_{1}\left(\frac{K^{2}}{4+2 K^{2}}\right)
$$

where $J_{n}$ are the Bessel functions of the first kind.

At the end of the laser heater, the periodic modulation amplitude will be washed out and the amplitude $A(s)$ will represent a smooth increase in the beam's energy spread. For a laser heater with uniform transverse profile, the slicewise energy modulation will follow an arcsin distribution, yielding a probability density like:

$$
f_{0}(s, \eta)=\frac{1}{\pi} \frac{1}{\sqrt{A(s)^{2}-\eta^{2}}},
$$

where $\eta=\left(\gamma-\gamma_{0}\right) / \gamma_{0}$ is the normalized energy variable. For more complicated transverse profiles [36,37], one must make suitable adjustments to the slice-wise energy spread introduce by the heater. In our expression for $f$, we have implicitly chosen an ideal beam which is infinite in $s$ and lacks a time-energy correlation. We will write the initial current profile as a flattop current of $I_{0}$.

After modulation by the lumped-laser heater element, we consider the effect of a bunch compressor. The linear optics can be accounted for changing coordinates to follow the particle trajectories as in $s=s_{0}+R_{56} \eta_{0}$, where $\left(s_{0}, \eta_{0}\right)$ are the initial coordinates, $(s, \eta)$ are the final coordinates, and $R_{56}$ is the chromatic dispersion. Following Liouville's theorem, the phase-space distribution after the dispersive section is given by

$$
f(s, \eta)=f_{0}\left(s_{0}, \eta_{0}\right)=f_{0}\left(s-R_{56} \eta, \eta\right) .
$$

Then, the current will be given by computing the marginal distribution in $s$, 


$$
I(s)=I_{0} \int f_{0}\left(s-R_{56} \eta, \eta\right) d \eta
$$

It will be convenient to work with the Fourier transform of the current, given by

$$
\begin{aligned}
\mathcal{F}[I](k) & =I_{0} \int e^{-i k\left(s+R_{56} \eta\right)} f_{0}(s, \eta) d s d \eta \\
& =I_{0} \int e^{-i k s}\left(\int e^{-i k R_{56} \eta} f_{0}(s, \eta) d \eta\right) d s .
\end{aligned}
$$

The inner integral is the Fourier transform of an arcsin distribution, which can be formally evaluated as the Bessel function $J_{0}\left(k R_{56} A(s)\right)$, however, to proceed analytically it is helpful to expand the exponential in a power series about $R_{56}=0$. Then, we will have a series involving moments of the modulation amplitude: $\left\langle A^{n}(s)\right\rangle=\int \eta^{n} f_{0}(s, \eta) d \eta$. For any symmetrical distribution, the odd moments vanish by parity and for arcsin the remaining sum can be written as

$$
\mathcal{F}[I](k)=I_{0} \int d s e^{-i k s} \sum_{n=0}^{\infty}\left(\frac{i R_{56}^{n}}{2^{n} n !}\right)^{2}(i k)^{2 n} A^{2 n}(s) .
$$

Finally, we can invert the Fourier transform term by term in the series,

$$
I(s)=I_{0} \sum_{n=0}^{\infty}\left(\frac{R_{56}^{n}}{2^{n} n !}\right)^{2} \frac{\partial^{2 n}}{\partial s^{2 n}} A^{2 n}(s) .
$$

Equation (1) gives our most intuitive picture of how laser heater shaping works. The $n=0$ term gives the unmodified current and the $n=1$ term gives the leading order modulation. The amplitude of the $n=1$ term immediately shows us that, to leading order, the bunching is proportional to the laser power $\left(A_{0}^{2}\right)$, rather than the electric field $\left(A_{0}\right)$. This reflects the indirect nature of bunch shaping via laser heater: linear contributions are washed out by the symmetry of the energy spread. The remaining first-order current profile is shaped like the second derivative of the laser power, such that a local maxima of the energy spread is transformed into a current minima, flanked by two smaller current peaks. Higher order terms sharpen the peaks and saturate the valley (which, after all, cannot go below zero current). Total charge is preserved at each order.

The expression we have used for $A(s)$ assumes that arbitrarily fine features can be written into the beam energy spread by the laser heater. In reality, the slippage dynamics we neglected within the wiggler set a limit of the modulation bandwidth: $\Delta \omega \ll \omega_{r} / N_{u}$, where $\omega_{r}$ is the resonant frequency and $N_{u}$ is the number of periods in the undulator. In practice, the slippage is rarely a matter for practical concern, both because most wigglers are short $\left(N_{u} \approx 10\right)$, and because (for reasonable modulation amplitudes) the beam's intrinsic energy spread will limit the bunching factor at high frequencies.

In order to see this, we can generalize Eq. (1) to the more realistic case in which the initial distribution has a Gaussian slice energy spread $\sigma_{\eta}$ convolved with the arcsin distribution. We also include a linear chirp $h$ which will compress the entire beam by a factor of $C=\left(1+h R_{56}\right)^{-1}$. The result is

$$
I(s)=C I_{0} \sum_{n=0}^{\infty}\left[\left(\frac{R_{56}^{n}}{2^{n} n !}\right)^{2} \frac{\partial^{2 n}}{\partial s^{2 n}}\left(A^{2 n}(C s) \circledast \frac{e^{-\frac{s^{2}}{2\left(R_{56} \sigma_{\eta}\right)^{2}}}}{\sqrt{2 \pi} R_{56} \sigma_{\eta}}\right)\right] .
$$

The chirp gives an overall scaling, while the incoherent energy spread is only important if $R_{56} \sigma_{\eta}$ is comparable to the length scale of the laser heater modulation. The energy spread becomes increasingly important when compared to higher order terms in the series, especially as we increase $R_{56}$ to approach full compression. For a modulation of magnitude $A_{0}$ occurring over a characteristic time scale $\sigma_{t}$, we can expect that the chromatic dispersion required for full compression would be $R_{56} \approx c \sigma_{t} / C A_{0}$. For these parameters, Eq. (1) suggests the incoherent energy spread will be unimportant if $\sigma_{\eta} \lesssim A_{0}$.

However, it is precisely as we approach full compression that the series solution presented here begins to require a large number of terms to reach convergence. This happens because as the phase space folds back on itself and begins to filament, the current spikes stop growing and begin changing shape. Thus, the limit $\sigma_{\eta} \lesssim$ $A_{0}$ applies only for the first term in the series, and near full compression we must always take $\sigma_{\eta}$ into account to determine the shape of the current spike. In many cases, this is actually a useful way to operate the heater, since the overheated, over-compressed electrons can be used to prevent undesired current spikes from forming at the edges of the beam. Nonetheless, the series solution gives a clear picture of how coherent microbunching arises from a time-dependent energy spread, and it shows how the laser heater parameters may be used to control the longitudinal phase space of the bunch.

\section{A. Gaussian}

In this section, we consider specialization to a single pulse with a Gaussian power profile:

$$
A(s)=A_{0} e^{-\frac{1 s^{2}}{c^{2} \sigma_{t}^{2}}}
$$

In this case, the convolution in Eq. (2) can be easily evaluated. Identifying $\tilde{\sigma}_{n}^{2}=c^{2} \sigma_{t}^{2} / n C^{2}+R_{56}^{2} \sigma_{\eta}^{2}$, we can write 
(a)

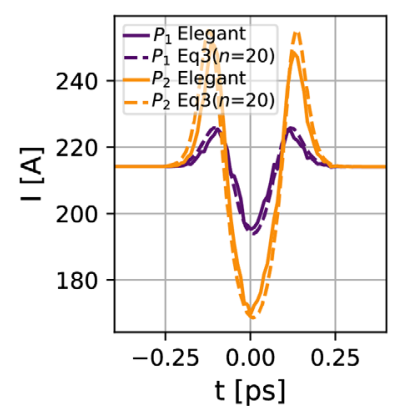

(b) $A_{0}$ Modulation [keV]

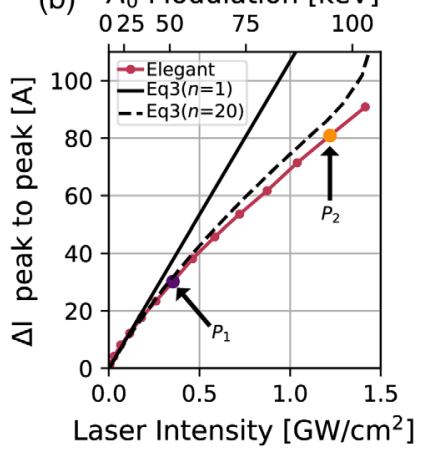

FIG. 2. Comparison of analytical model with ELEGANT simulation of a laser heater plus bunch compressor. (a) Current profiles for small and large laser power. At large power, multiple terms are required for the series solution to converge. (b) Peak to peak current modulation as a function of laser intensity, with callouts for the points shown to the left.

$$
\begin{aligned}
I(s)= & C I_{0} \sum_{n=0}^{\infty}\left[\left(\frac{R_{56} A_{0}}{2 \tilde{\sigma}_{n}}\right)^{2 n} \frac{c \sigma_{t}}{C \sqrt{n} \tilde{\sigma}_{n}} \frac{(2 n) !(-1)^{n}}{2^{n}(n !)^{3}}\right. \\
& \left.\times\left(\frac{\tilde{\sigma}_{n}^{2 n} 2^{n}(n !)}{(2 n !)(-1)^{n}}\right) \frac{\partial^{2 n}}{\partial s^{2 n}}\left(e^{-\frac{1 s^{2}}{2 \tilde{\sigma}_{n}^{2}}}\right)\right],
\end{aligned}
$$

where the second line has been written such that it evaluates to 1 at $s=0$ for all $n$ and thus the sum of the top line alone gives the current at the $t=0$ minimum.

In Fig. 2, we evaluate Eq. (3) for parameters similar to the LCLS-II CuS line: $R_{56}=0.55 \mathrm{~mm}, C=5.2, \gamma_{0}=$ $215 \mathrm{MeV}$, and $\sigma_{\eta}=20 \mathrm{keV} / \gamma_{0}$ for a variety of laser heater amplitudes, all with field full width at half maximum (FWHM) 1 ps (i.e., power FWHM $1 / \sqrt{2}$ ps). We compare the analytical result to simulations in ELEGANT [38]. At low intensity both relations are linear, while at higher intensity the current modulation begins to saturate and more terms are needed to make Eq. (3) converge. In this example, we can use just one term up to a $10 \%$ current modulation, but soon after we need as many as 20 terms (for $I_{s}<1.4 \mathrm{GW} / \mathrm{cm}^{2}$ ). The remaining discrepancy between model and simulation can be attributed to the fact that the ELEGANT lattice includes a realistic beamline with rf curvature and nonlinear optics.

\section{B. Pulse stacking}

A quasiperiodic pulse train can be built by stacking consecutive Gaussian laser heater pulses with some time delay $\Delta t$. Lasing with such a current profile will create persistent temporal and spectral features that can be manipulated for use with correlation imaging techniques $[39,40]$. We build a simple description showing how control of a laser pulse stacker translates to control over the beam bunching factor.

When the separation between pulses is large compared to their extent $\left(\Delta t \gg c \sigma_{t}\right)$, we can simply superimpose copies of the current modulation in Eq. (3). This covers most practical arrangements of a pulse stacker, since closely

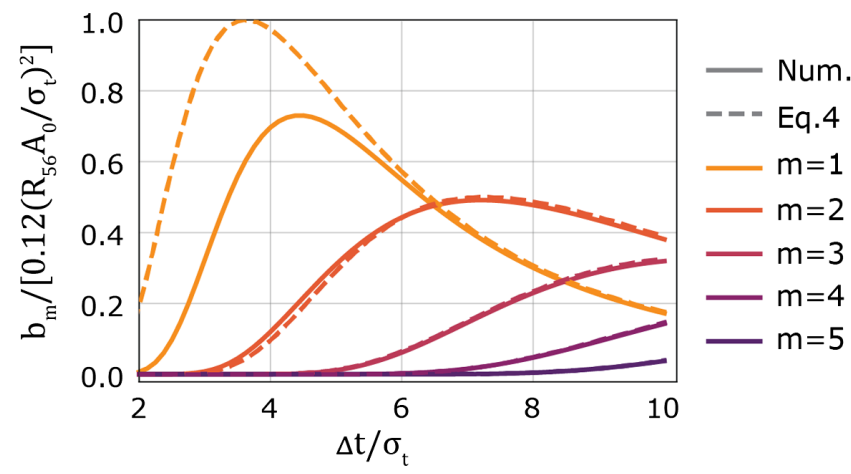

FIG. 3. Magnitude of the bunching factor for a train of Gaussian laser heater pulses as a function of pulse spacing. Dashed lines are from Eq. (4), while solid lines are from a numerical integration of the Vlasov equation, taking into account cross talk between neighboring pulses.

spaced Gaussians will overheat and spoil the entire beam. For an infinite stack of identical Gaussian pulses separated in time by $\Delta t=2 \pi / k_{0} \gg c \sigma_{t}$, we find the bunching factor at frequency $m k_{0}$ to be

$b_{m}=\sum_{n=0}^{\infty}\left[k_{0}\left(m k_{0} R_{56} A_{0}\right)^{2 n} \frac{c \sigma_{t}}{C \sqrt{n}} \frac{(-1)^{n}}{2^{2 n}(n !)^{2}}\left(e^{-\frac{1}{2}\left(m k_{0}\right)^{2} \tilde{\sigma}_{n}^{2}}\right)\right]$.

To order $O\left(A_{0}^{2}\right)$ and for $\sigma_{\eta} \approx 0, b_{m}$ is maximized when the spacing between Gaussian peaks is $\Delta t=$ $2 \pi m c \sigma_{t} / \sqrt{3}$. For $b_{1}$, this roughly corresponds to placing the current maxima in Fig. 2(a) halfway between the successive minima, while the harmonics are maximized by spacing the pulses farther apart. At this maximum $\left|b_{1}\right| \approx 0.12\left(R_{56} A_{0} / \sigma_{t}\right)^{2}, b_{1}$ is the largest of the harmonics.

We can see this behavior in Fig. 3, where we have plotted the bunching factor for a train of Gaussian pulses as a function of spacing. All pulses have $\sigma_{\eta}=0, C=1$, and we use $R_{56} A_{0} / \sigma_{t} \ll 1$ such that Eq. (4) is well represented by $n=1$. By comparing Eq. (4) to a numerical calculation (directly integrating $\int f_{0}\left(s-R_{56} \eta\right) d \eta$, were $f_{0}$ contains the modulation from the Gaussian pulse train), we can see that at small spacing Eq. (4) overestimates the bunching, because it does not consider the energy spread induced by neighboring pulses. Nonetheless, it provides a useful way to estimate the bunching factor after the first stage of compression.

\section{Pulse train}

Higher frequency, more strongly periodic current modulations can be created by the chirped pulse beating technique [41,42]. Such a beam has drawn interest in mode-locking schemes, as well as for control of sidebands and other quasicoherent features [43].

A perfectly sinusoidal power profile is a particularly simple case to analyze. For a spatial wavelength $2 \pi / k_{0}$ : $A(s)=A_{0} \sin \left(k_{0} s\right)$ we find, analogous to [28], but for an 
unmatched laser spot (and arcsin energy distribution), we can derive the exact result:

$$
\mathcal{F}[I](k)=I_{0} \sum_{m} b_{2 m} \delta\left(k+2 m k_{0}\right),
$$

where the bunching factor is given by

$$
b_{2 m}=\left(J_{m}\left(n k_{0} R_{56} A_{0}\right)\right)^{2} e^{-2 m^{2} k_{0}^{2} R_{56}^{2} \sigma_{\eta}^{2}} .
$$

As expected from Eq. (2), the bunching depends on $A_{0}^{2}$ to lowest order. But, we can also see that as $k A_{0} R_{56}$ is increased, the initial bunching at $b_{2}$ will begin to saturate and be replaced by higher order bunching terms.

\section{Staged microbunching evolution of current spikes from heater shaping}

The proceeding analysis describes how a single bunch compressor translates an energy modulation into a current modulation. But in a realistic beamline, the appearance of a current modulation will begin to drive collective forces and feedback into microbunching gain.

Unlike the traditional analysis of microbunching gain, here we have to consider a fully coherent modulation. That is, the filamentation of phase space following compression leads to a complicated time-energy structure which can damp (or enhance) microbunching.

Despite these complications, We can gain some appreciation for the effect of staging by considering the space charge impedance [19,44]:

$$
Z_{l s c}=\frac{i Z_{0}}{\pi k r_{b}^{2}}\left[1-\frac{k r_{b}}{\gamma} K_{1}\left(\frac{k r_{b}}{\gamma}\right)\right] \approx \frac{i Z_{0}}{4 \pi \gamma^{2}} k \ln \left(\frac{2 k r_{b}}{\gamma}\right)^{2}
$$

where $K_{1}$ is a modified Bessel function of the second kind, $Z_{0}$ is the impedance of free space, $r_{b}$ is the radius of the electron beam, and the second half shows the limiting form for $k r_{b} / \gamma \ll 1$. This impedance gives us the Fourier transformed electric field in terms of the Fourier transform of the current: $E(s)=\mathcal{F}^{-1}\left[Z_{l s c} \mathcal{F}[I(s)]\right]$. In order to approximate the inverse Fourier transform, we assume the beam current spectrum falls off rapidly above a characteristic frequency defined as $k_{\max } \approx 1 / \sigma_{z}$. Then, provided that the limiting form holds, we can approximate the energy chirp due to longitudinal space charge by subtracting from the impedance a term proportional to $k \log \left(\sqrt{2} \sigma_{z} k\right)$ [45], which we can then neglect since it is small below the cut off frequency. We are left with an impedance $\propto k$, such that the energy chirp is simply

$$
\partial_{z} \gamma \approx \frac{1}{\gamma^{2}} \ln \left(\frac{8 \gamma^{2} \sigma_{z}^{2}}{r_{b}^{2}}\right) \partial_{s}\left(\frac{I(s)}{I_{A}}\right),
$$

where $I_{A}=4 \pi \epsilon_{0} m c^{3} / q_{e} \approx 17 \mathrm{kA}$ is the Alfven current. This chirp goes like the derivative of the current profile, such areas near a current maxima will become chirped. Subsequent regions with anomalous chromatic dispersion (e.g., the natural $R_{56}$ in a dogleg) will recompress such a chirp into an even larger current maxima. This creates a feedback cycle which can amplify small, high frequency, modulations into large amplitude current spikes.

The gain becomes quite complicated in cases where the space-charge induced energy spread is comparable to the laser heater induced energy spread. In this case, the fully coherent problem needs to be analyzed. In a real beamline, one also has to consider the role of other collective effects (namely the linac wakefield and coherent synchrotron radiation) in asymmetrically chirping the bunch. In these cases, it quickly becomes expedient to pursue a fully numerical, particle tracking-based approach.

\section{NUMERICAL STUDY OF CURRENT SPIKES}

Here, we present a series of numerical studies at the LCLS-II beamlines, which are represented in a block-diagram format in Fig. 4(I). LCLS-II has both a superconducting $(\mathrm{sc})$ beamline and a normal conducting (copper, cu) beamline, each of which can be sent to either the soft $\mathrm{x}$-ray undulators (S) or the hard $\mathrm{x}$-ray undulators $(\mathrm{H})$, leading to the four possibilites: $\mathrm{scS}, \mathrm{scH}, \mathrm{cuH}$, and $\mathrm{cuS}$. We specialize to the two soft $\mathrm{x}$-ray configurations, $\mathrm{scS}$ and cuS. Both beamlines consist of an injector followed by a laser heater and two bunch compression stages, before the beam switch yard routes the beam to its final destination.

The high-level similarities in beamline layout belie major differences in beam dynamics. These differences are hinted at in Fig. 4(II) where we show the beam energy vs $\mathrm{z}$ for the two beamlines. The superconducting linac accelerates at a lower gradient, has an extra $1.5 \mathrm{~km}$ of transport, and a 6x larger net compression compared to the copper case. This is partially compensated for by operating at a lower peak current, but the end result is that the
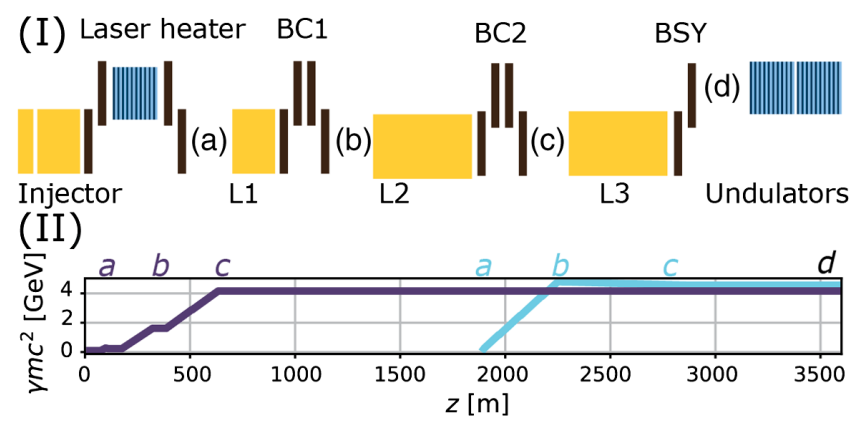

FIG. 4. (I) Cartoon of LCLS-II beamlines (not to scale). "L" stands for linac, "BC" for bunch compressor, and "BSY" for beam switch-yard. The BSY is a sequence of doglegs which nominally have $R_{56}=0$ but may be adjusted to allow lower energy particles to move toward the head of the bunch. (II) Central energy along the superconducting (purple) and copper (light blue) beamlines, illustrating the vast differences in space charge impedance in the two cases. 
superconducting beam experiences larger space charge impedance and larger microbunching gain than the copper beam. Consequently, despite the fact that both beamlines have very similar BC1s, we will use a weaker laser heater modulation on the superconducting line.

We will explore how the laser-heater shapes the beam dynamics in these two cases by studying the output of startto-end simulations at the locations marked a,b,c,d in Fig. 4. Our simulations are performed in ELEGANT [38], based on the LCLS-II lattices [46] using beams from dedicated injector simulations. This method has been successfully benchmarked at LCLS $[47,48]$, and includes models for linac wakefields, longitudinal space charge, and coherent synchrotron radiation in addition to standard (nonlinear) optical maps for the static beamline elements.

The simulations shown here are run with more than 1 million particles (up to 100 million for simulations sensitive to high-frequency microbunching gain), and for most elements requiring the beam profile we use between 200 and 500 bins implying a cut off frequency near $0.5 \mathrm{Phz}$ (for the fully compressed bunch). However, when the beam shaping introduces strong high-frequency components (as in the case of the beatwave), we must increase the sampling rate to capture the collective dynamics. For the laser heater, we use the "LSRMDLTR" element with a custom power profile for the laser, while assuming the reference particle meets the resonance condition. In addition to the shaped laser pulses, we superimpose a weak, flattop, power profile which contributes a uniform energy spread to suppress unwanted high-frequency microbunching.

\section{A. Single spike}

An isolated current spike can be used to generate attosecond $\mathrm{x}$-ray pulses by the chirp-taper technique [10]. The current spikes we show here could be used to lase directly, or they could first be used to drive CSR in a wiggler in direct analogy to Ref. [10]. In either case, the goal is to create a high contrast, few-femtosecond current spike.

A single Gaussian laser heater, like that from Sec. II A, will create two current spikes after $\mathrm{BC} 1$. To turn this into a single, isolated, spike we shift the laser heater such that one of the current spikes forms near the head of the bunch, where the current is lower. The microbunching gain will be larger for the trailing spike, which lies next to a cold, fresh beam ready to be modulated by the space-charge impedance. This allows us to form an isolated few kA spike after the second stage of bunch compression. The spike can be further enhanced by anomalous chromatic dispersion $\left(R_{56}\right)$ in the BSY.

In Fig. 5, we illustrate this process through simulation of the LCLS-II cuS beamline. By using the laser parameters recorded in Table I, we introduce a large energy spread, visible in panel (a) as a Gaussian protrusion at $t \approx-1 \mathrm{ps}$ distinct from the uniform background heating. The modulation strength is made large enough to maximize the (a)

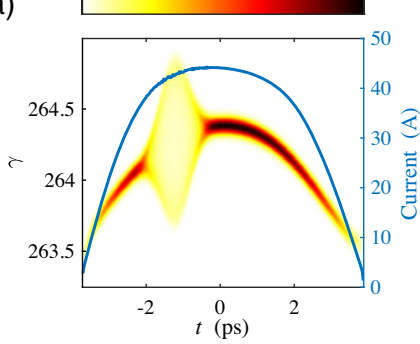

(c)
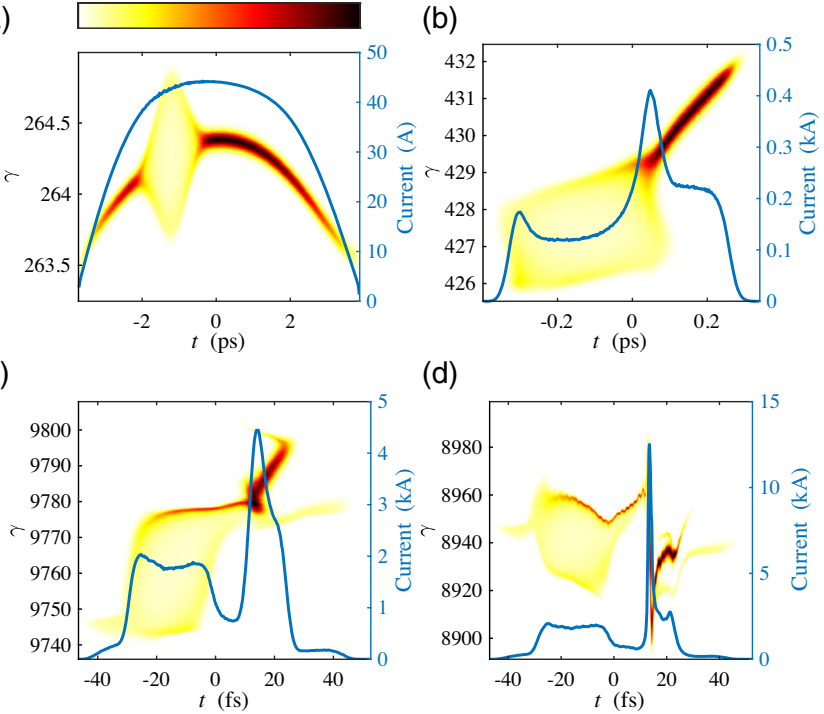

(d)

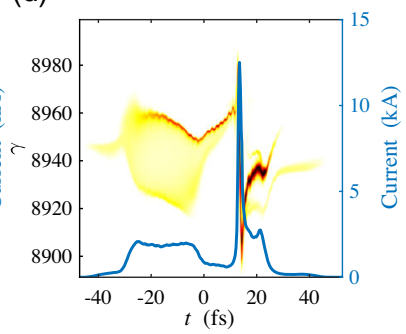

FIG. 5. Generation of a single high-current spike at the LCLS-II cuS beamline. Longitudinal phase space: (a) after laser heater; (b) after the first bunch compressor; (c) after the second bunch compressor; (d) at the entrance of soft x-ray undulator. The colorbar above (a) shows the relative density for each plot. See Table I.

current spikes which form after $\mathrm{BC} 1$, as shown in panel (b) The spike at $t=0 \mathrm{ps}$ exceeds that at $t=-0.3 \mathrm{ps}$ because the initial current density at $t=0$ is larger. Between panels (b) and (c), the space charge impedance introduces a local chirp along the bunch. This chirp is most pronounced, relative the slice energy spread, near the tail of the bunch, and so the second bunch compressor creates a single large spike in panel (c). Between (c) and (d), there is again a long transport with large space charge impedance, leading to a strongly chirped bunch which is further compressed by the anomalous chromatic dispersion $\left(R_{56}\right)$ of the BSY. At the entrance of the soft x-ray undulator, we can get a high current $(>10 \mathrm{kA})$ and short $(\sim 1.5 \mathrm{fs})$ spike in the beam.

TABLE I. Laser heater shaping parameters to generate a single high-current spike at the LCLS-II cuS beamline.

\begin{tabular}{lc}
\hline \hline Beamline & cuS \\
\hline Number of stacked Gaussians & 1 \\
Pulse width $\tau$ & $0.95 \mathrm{ps}$ \\
Pulse power $P$ & $60 \mathrm{MW}$ \\
Laser waist $w_{0}$ & $600 \mu \mathrm{m}$ \\
Electron size $(\mathrm{rms}) \sigma_{r}$ & $140 \mu \mathrm{m}$ \\
Peak energy spread $A_{0}$ & $275 \mathrm{keV}$ \\
Uniform energy spread & $20 \mathrm{keV}$ \\
BC1 $R_{56}$ & $-45.7 \mathrm{~mm}$ \\
BC1 compression factor $C_{1}$ & 5.2 \\
BC2 $R_{56}$ & $-28.6 \mathrm{~mm}$ \\
BC2 compression factor $C_{2}$ & 10.5 \\
\hline \hline
\end{tabular}



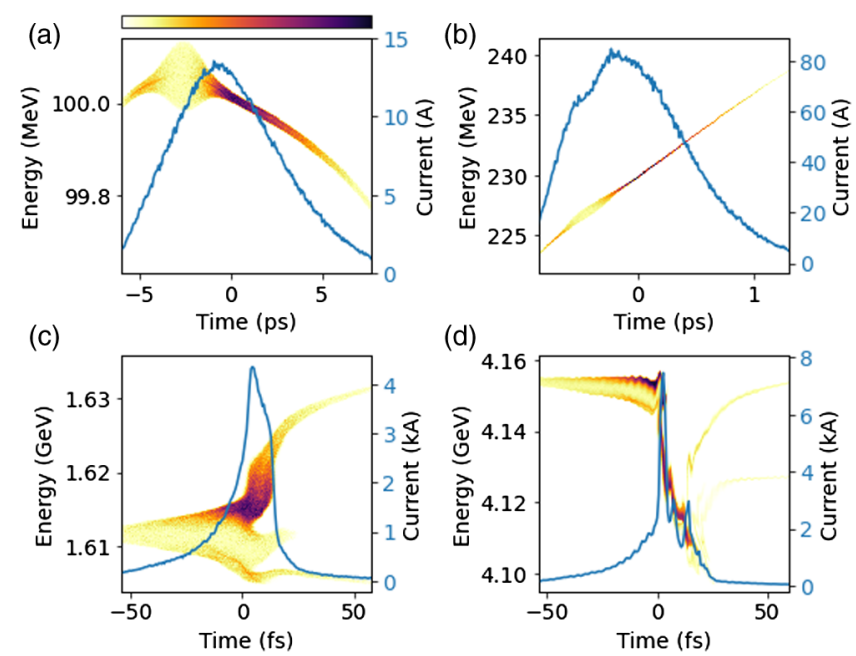

FIG. 6. Generation of a single high-current spike at the LCLS-II scS beamline. Longitudinal phase space: (a) after laser heater; (b) after the first bunch compressor; (c) after the second bunch compressor; (d) at the entrance of soft x-ray undulator. The colorbar above (a) shows the relative density for each plot. See Table II.

Next, we can consider a similar approach applied to the LCLS-II scS line (Fig. 6 and Table II). Here, we use a wider laser heater pulse (centered at $t=-4 \mathrm{ps}$ in panel a), which creates a much smaller modulation (near $t=-0.5 \mathrm{ps}$ ) after $\mathrm{BC} 1$ (panel b) than we saw in the cuS beamline. This is appropriate because the sc beamline has lower energy and larger space-charge impedance between $\mathrm{BC} 1$ and $\mathrm{BC} 2$. As a result, this small modulation is enough to create an isolated current spike near the tail of the bunch after the large compression factor in the $\mathrm{BC} 2$ chicane (c). This beam can still be successfully transported through the bypass line and the BSY to the SXR undulators.

\section{B. Pulse stacking}

Here, we consider creating a quasiperiodic bunch train by stacking four Gaussian pulses on top of the regular $\mathrm{LH}$

TABLE II. Laser heater shaping parameters to generate a single high-current spike at the LCLS-II scS beamline.

\begin{tabular}{lc}
\hline \hline Beamline & $\mathrm{scS}$ \\
\hline Number of stacked Gaussians & 1 \\
Pulse width $\tau$ & $1.5 \mathrm{ps}$ \\
Peak power $P$ & $1.44 \mathrm{MW}$ \\
Laser waist $w_{0}$ & $180 \mu \mathrm{m}$ \\
Electron size $(\mathrm{rms}) \sigma_{r}$ & $130 \mu \mathrm{m}$ \\
Peak energy spread $A_{0}$ & $70 \mathrm{keV}$ \\
Uniform energy spread & $12 \mathrm{keV}$ \\
BC1 $R_{56}$ & $-53 \mathrm{~mm}$ \\
BC1 compression factor $C_{1}$ & 6.3 \\
BC2 $R_{56}$ & $-40.4 \mathrm{~mm}$ \\
BC2 compression factor $C_{2}$ & 51 \\
\hline \hline
\end{tabular}
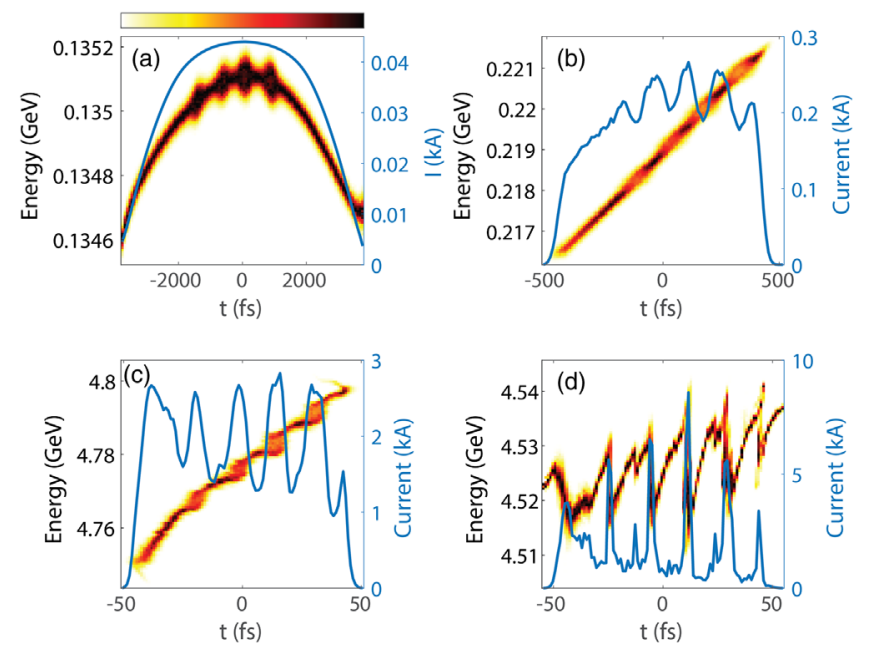

FIG. 7. Generation of a short bunch train at the LCLS-II cuS beamline. Longitudinal phase space: (a): after the laser heater, (b): after BC1, (c): after BC2, and (d) in the middle of soft x-ray self seeding chicane, with an effective $R_{56}$ of $0.3 \mathrm{~mm}$. The colorbar above (a) shows the relative density for each plot. See Table III.

pulse, as described in Sec. II B. Such a pulse stacking arrangement can be created, for example, by sending a single Gaussian laser pulse of (power) FWHM 0.47 ps into a double interferometer that replicates the original Gaussian laser pulse into four copies. We can then tune the relative strength of each pulse with a polarizer and a waveplate, as well as the separation between the pulses by changing a time delay. This flexibility turns out to be necessary in order to compensate long-range wakefield effects and balance the compression of the resulting current spikes.

In Fig. 7, we show a simulation of this bunch stacker at the LCLS-II cuS beamline. Each of the four Gaussian pulses creates two current maxima, but in-between pulses these maxima are made to overlap such that the fundamental bunching is maximized. Thus, we end up with six current spikes, where the first and last spike are roughly

TABLE III. Laser heater shaping parameters to generate a short bunch train at the LCLS-II cuS beamline.

\begin{tabular}{lc}
\hline \hline Beamline & $\mathrm{cuS}$ \\
\hline Number of stacked Gaussians & 4 \\
Pulse width $\tau$ & $0.47 \mathrm{ps}$ \\
Pulse separation & $0.8,0.7,0.8 \mathrm{ps}$ \\
Peak powers $P$ & $0.8,0.8,1,1 \mathrm{MW}$ \\
Laser waist $w_{0}$ & $450 \mu \mathrm{m}$ \\
Electron size (rms) $\sigma_{r}$ & $140 \mu \mathrm{m}$ \\
Peak energy spread $A_{0}$ & $40,50,50 \mathrm{keV}$ \\
Uniform energy spread & $20 \mathrm{keV}$ \\
BC1 $R_{56}$ & $-45.7 \mathrm{~mm}$ \\
BC1 compression factor $C_{1}$ & 5.2 \\
BC2 $R_{56}$ & $-22.7 \mathrm{~mm}$ \\
BC2 compression factor $C_{2}$ & 9.4 \\
\hline \hline
\end{tabular}



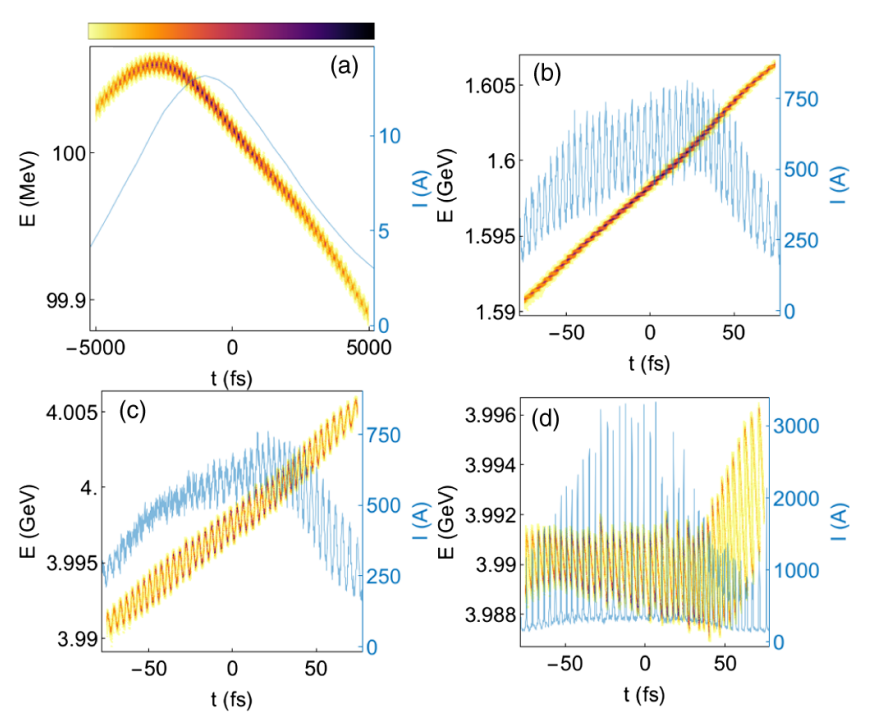

FIG. 8. Generation of highly periodic bunch train at the LCLS-II scS beamline. Longitudinal phase space: (a) after the laser heater, (b) after BC2, (c) after the dogleg, and (d) at the center of the soft x-ray undulator entrance. The colorbar above (a) shows the relative density for each plot. See Table IV.

half-strength. In order to finish with all spikes at full compression, we make the first two pulses $20 \%$ weaker than the last two (see Table III). Additionally, we note that the current spikes shown in panel (d) are located after a selfseeding chicane, such that the current spikes only become fully compressed halfway through the undulator line. This allows the spikes to be used in a self-seeding scheme and thus to create a set of discrete spectral sidebands for use in correlation spectroscopy [39].

\section{Pulse train}

Here, we consider creating a high frequency, periodic current modulation using an intensity modulated pulse on top of the regular LH pulse, as described in Sec. II C. The intensity modulation can be generated using the chirp pulse beating technique [41,42]. Here, a single temporally stretched Gaussian laser pulse with a linear chirp is duplicated in an interferometer. Introducing a variable time delay to one pulse and overlapping both temporally produces a constant frequency difference proportional to the time delay and chirp, resulting in the desired intensity modulation.

In Fig. 8, we show a simulation of this current modulation at the LCLS-II scS beamline. The simulated $50 \mu \mathrm{m}$ intensity modulation assumes a laser pulse with $10 \mathrm{~nm}$ Fourier limited bandwidth, stretched to $20 \mathrm{ps}$ with a $19.85 \mathrm{ps}$ delay between split pulses, Fig. 8(a). Additional simulation parameters are shown in Table III. Here, the peak energy spread introduced in the laser heater is equal to the nominal heating for suppressing the microbunching instability in nominal LCLS-II operations. This energy modulation is small compared to the previous cases and will not generate significant density modulation until after $\mathrm{BC} 2$, producing an
TABLE IV. Laser heater shaping parameters to generate a highly periodic bunch train at the LCLS-II $\mathrm{scS}$ beamline. See Fig. 8.

\begin{tabular}{lc}
\hline \hline Beamline & $\mathrm{scS}$ \\
\hline Beatwave modulation period & $50 \mu \mathrm{m}$ \\
Final modulation period & $1.2 \mu \mathrm{m}$ \\
Peak power $P$ & $0.48 \mathrm{MW}$ \\
Laser waist $w_{0}$ & $2600 \mu \mathrm{m}$ \\
Electron size $(\mathrm{rms}) \sigma_{r}$ & $130 \mu \mathrm{m}$ \\
Peak energy spread $A_{0}$ & $6 \mathrm{keV}$ \\
Uniform energy spread & $2 \mathrm{keV}$ \\
BC1 $R_{56}$ & $-55 \mathrm{~mm}$ \\
BC1 compression factor $C_{1}$ & 2.75 \\
BC2 $R_{56}$ & $-70.5 \mathrm{~mm}$ \\
BC2 compression factor $C_{2}$ & 18.5 \\
\hline \hline
\end{tabular}

approximately sinusoidal current modulation with a compressed period of $1.2 \mu \mathrm{m}$, Fig. 8(b). As the beam traverses L3, this density modulation leads to a sinusoidal energy modulation driven by longitudinal space charge (to accurately capture this modulation in elegant simulations the number of bins and high-frequency cutoff are adjusted to encompass frequencies up to the third harmonic of the compressed modulation period). This energy modulation can be controlled by adjusting the $\mathrm{BC} 2$ compression factor and/or increasing the baseline (flattop) laser heater pulse. Furthermore, in order to produce an approximately uniform, periodic modulation across the beam, we consider the use of octupoles to reduce nonlinear compression as was proposed in Ref. [49].

In order to reduce LSC energy modulation in the long bypass line, anomalous chromatic dispersion $\left(R_{56}\right)$ is introduced by the dogleg located immediately downstream of L3. Here the total $R_{56}$ can be tuned to minimize the current modulation by adjusting compensating chicanes at the dogleg entrance and exit, Fig. 8(c). Finally, this energy modulation is converted to density modulation by the selfseeding chicane located in the middle of the SXR line, Fig. 8(d). This allows the spikes to be used in self-seeding schemes as described in the previous section.

\section{HORN SUPPRESSION}

During compression of a long electron bunch, nonlinear optics generate caustics, typically in the form of two current spikes at the head and tail of the bunch [50]. These current spikes, colloquially known as "horns", can induce CSR kicks to the beam and significantly increase its projected transverse emittance [51].

In the LCLS, these horns limit the efficiency of standard SASE FEL lasing. To mitigate this effect, the LCLS employs collimators in a dispersive region of the first bunch compressor to remove the parts of the electron beam affected by this third order chirp [8]. This approach is not 
suitable for high-average power machines such as the LCLS-II superconducting linac or the European XFEL, where the high-beam power would damage the collimators. Consequently, one must pursue new approaches, such as using compensatory nonlinear optics [49].

On the other hand, advanced short pulse FEL experimental setups utilize these current horns to modulate the electron beam [10,52]. Thus, it is desirable to have a horn suppression mechanism which can operate with Mhz flexibility in order to interleave beams with and without horns for downstream distribution to separate users. This can be accomplished by programmaticlly switching a shaped laser heater pulse into the beamline.

A shaped laser heater can suppress the horns by heating the edges of the bunch and decreasing the phase space density that will be mapped to the caustic. At the same time, the core of the bunch should not be over-heated, which implies that the heater pulse should be shaped concave upwards, like a parabola. The new energy spread will, inturn, create its own current spikes: from Eq. (1), we can predict that a parabolic laser heater will generate a parabolic current perturbation: $\partial_{s}^{2}\left(s^{2}\right)^{2}=12 s^{2}$. Design of an effective horn suppression requires balancing these two effects.

We illustrate the suppression mechanism in Fig. 9 for a simple case in which the caustic is created by a cubic chirp and suppressed by a parabolic laser heater: $\eta_{f}=\eta_{i}+\alpha t^{3} / \tau^{3}+f_{0}\left(A_{0}(t / \tau)^{2}\right)$. We also include a small incoherent energy spread $(0.1 \%)$. When the laser heater is turned off $(a, b)$ the cubic chirp leads to large, narrow current spikes with lots of high-frequency content. The laser heater $(\mathrm{c}, \mathrm{d})$ suppresses these horns by reducing the phase-space density which would be mapped to the caustic.

(a)

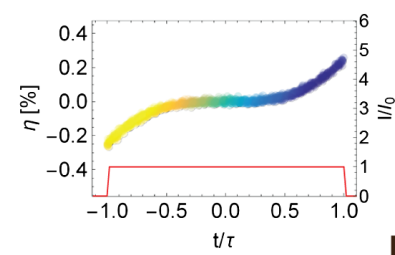

(c)

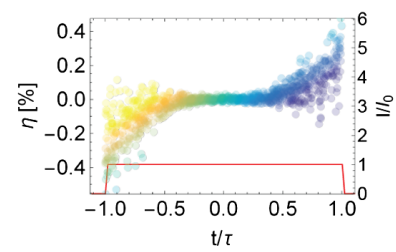

FIG. 9. Cartoon illustrating heater horn suppression. From (a) to (b) a chicane compresses a beam with cubic chirp forming a caustic. From (c) to (d) the same caustic is suppressed due to energy spread from a laser heater. Particles in frame (a)[b] are colored by final $t$ coordinate from (b)[d] such that we can see which parts of the initial phase space lead to the caustic. Current profiles are shown in red.
We cannot, however recover a flattop profile, since the heater generates its own parabolic profile near $t=0$ (recall Eq. (1) does not take into account edge effects). At any rate, both the peak current and the high-frequency content are greatly reduced such that the CSR and spacecharge impedances become significantly less disruptive. We explore this concept in more detail with full numerical simulations in the next section.

\section{A. Simulations}

As we did for the current spike generation, we will use ELEGANT [38] to model the beam dynamics associated with horn suppression at the LCLS-II cuS and scS beamlines. In place of a perfectly parabolic laser heater, we use a more practical arrangement of two Gaussian heater pulses. We also include a direct comparison to the case with the Gaussian heater pulses, in order to emphasize the difference between the caustics and heater-induced current modulation.

Figure 10 shows simulations of the LCLS Cu beamline for a $250 \mathrm{pC}$ bunch charge compressed to $3 \mathrm{kA}$. Horns formed within the second bunch compressor increase the projected horizontal emittance from 0.50 to $1.38 \mu \mathrm{m}$, and a subsequent CSR kick from the LCLS second dog leg leads to a final emittance of $2.78 \mu \mathrm{m}$ at the undulator line. Applying two Gaussian laser pulses each of duration 1 ps and delayed by $4 \mathrm{ps}$, heats the head by $190 \mathrm{keV}$ and the tail by $220 \mathrm{keV}$, dispersing the charge which would have otherwise formed the horns in the bunch compressor. This mitigates some of the CSR kick-induced emittance growth while producing a similar peak current. The resulting projected emittance after the second bunch compressor and undulator entrance is a more modest $0.70 \mu \mathrm{m}$. The reshaped current profile also alters the wakefield and space-charge induced beam chirp, such that in a multiplexed operation the two beams typically have different chirps (as shown).

The LCLS-II superconducting linac is positioned $2 \mathrm{~km}$ upstream of the copper linac, but shares the same FEL beamlines with the copper linac, necessitating the long transport line shown in Fig. 4(II). Current horns formed in the bunch compressor of the superconducting linac can induce strong wakefields along this long transport line, increasing the energy spread of the electron beam and degrading FEL lasing performance.

Simulations of the LCLS-II scS beamline are shown in Fig. 11 using the baseline scenario of a $100 \mathrm{pC}$ beam formed by a temporally flattop cathode laser. Increasing the baseline compression from $800 \mathrm{~A}$ to $2 \mathrm{kA}$ results in high-current horns and significant energy variation along the beam. Heating the head and tail of the beam with $1.1 \mathrm{ps}$ pulses spaced 7 ps apart results in a beam of similar duration (50 fs FWHM) and current but with 50\% less energy variation. Furthermore, several additional horizontal and vertical bends are needed to transport the 

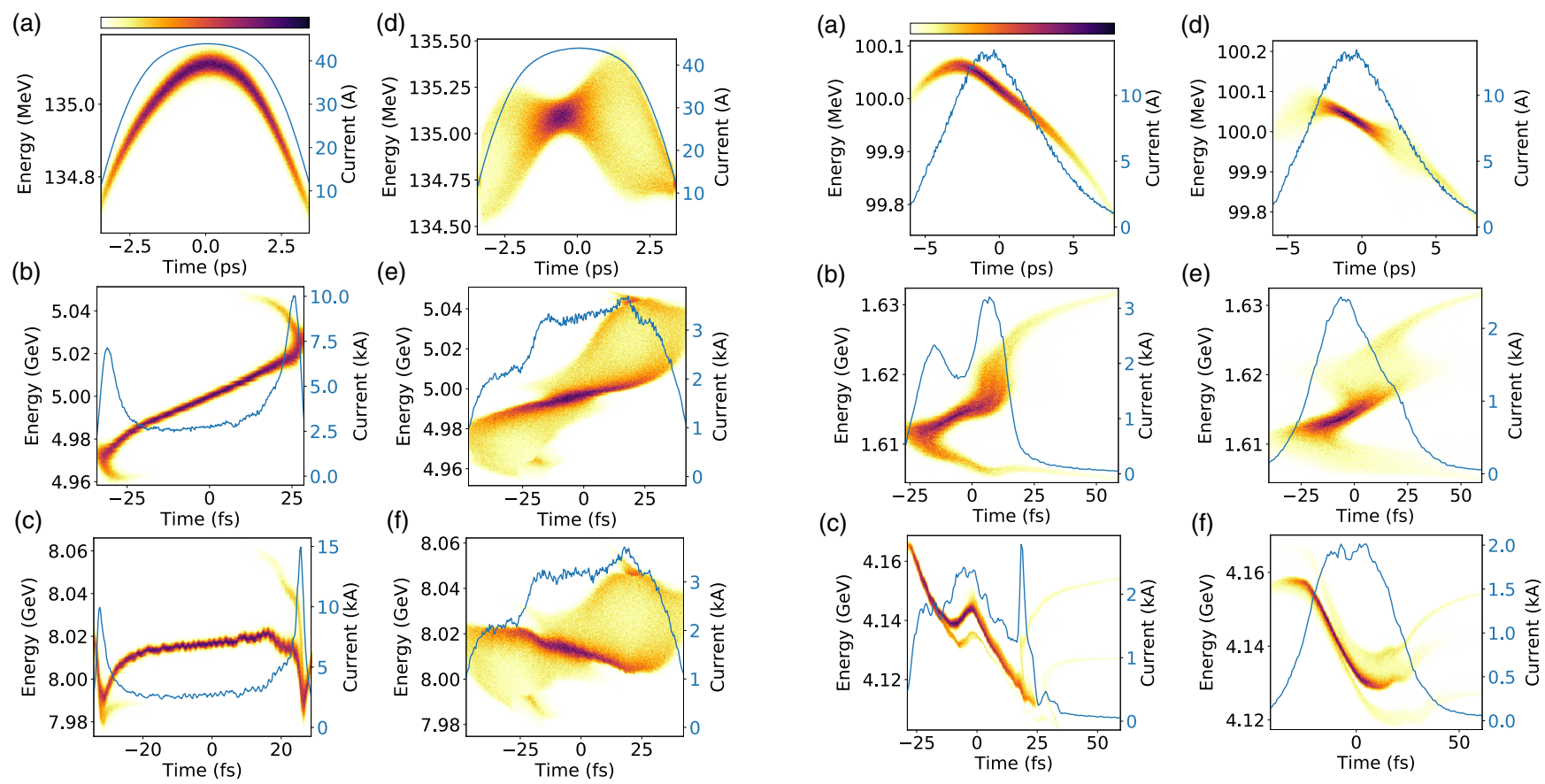

FIG. 10. Elegant simulations (a-c) without and (d-f) with laser heater horn collimation for the LCLS-II normal conducting, copper linac. Figures (a) and (d) show the longitudinal phase space after the laser heater with and without the pairs of Gaussian pulses. Figures (b) and (e) show the beam after the second bunch compressor. Figures (c) and (f) show the beam at the entrance of the hard x-ray undulators. The color bar above (a) shows the relative density for each plot. See Table V.

superconducting beam to the soft x-ray FEL line, each of which can spoil the emittance via CSR. The horizontal and vertical projected emittances at the undulator line are reduced from 2.68 and $0.66 \mu \mathrm{m}$ without laser heater shaping to 1.87 and $0.50 \mu \mathrm{m}$ with.

In both beamlines, we observe a large total energy spread caused by the heater, due both to the total chirp and to

TABLE V. Laser heater shaping parameters to suppress caustic formation at the LCLS and at the LCLS-II scS.

\begin{tabular}{lcc}
\hline \hline Beamline & LCLS & LCLS-II scS \\
\hline Number of stacked Gaussians & 2 & 2 \\
Pulse width $\tau$ & $1 \mathrm{ps}$ & $1.1 \mathrm{ps}$ \\
Pulse separation & $5.5 \mathrm{ps}$ & $7 \mathrm{ps}$ \\
Peak powers $P$ & $2,2.75 \mathrm{MW}$ & $200,200 \mathrm{~kW}$ \\
Laser waist $w_{0}$ & $280 \mu \mathrm{m}$ & $180 \mu \mathrm{m}$ \\
Electron beam size $\sigma_{x}$ & $140 \mu \mathrm{m}$ & $115 \mu \mathrm{m}$ \\
Peak energy spread $A_{0}$ & $120,180 \mathrm{keV}$ & $33,33 \mathrm{keV}$ \\
Uniform energy spread & $20 \mathrm{keV}$ & $8 \mathrm{keV}$ \\
BC1 $R_{56}$ & $-48.0 \mathrm{~mm}$ & $-52.5 \mathrm{~mm}$ \\
BC1 compression factor $C_{1}$ & 5.2 & 5.8 \\
BC2 $R_{56}$ & $-24.9 \mathrm{~mm}$ & $-40.3 \mathrm{~mm}$ \\
BC2 compression factor $C_{2}$ & 15.2 & 17.2 \\
\hline \hline
\end{tabular}

FIG. 11. Elegant simulations (a-c) without and (d-f) with laser heater horn collimation for the LCLS-II superconducting linac. Figures (a) and (d) show the longitudinal phase space after the laser heater with and without the pairs of Gaussian pulses. Figures (b) and (e) show the beam after the second bunch compressor. Figures (c) and (f) show the beam at the entrance of the soft x-ray undulators. The color bar above (a) shows the relative density for each plot. See Table V.

increased slice energy spread. The chirp can be reduced by changing the peak current and the compression ratio between $\mathrm{BC} 1$ and $\mathrm{BC} 2$ (when multiplexing, these changes become a compromise between the needs of the heater-on and heater-off users), but the slice energy spread is inherent to the technique. This is not a major impediment to lasing however, since the spread comes from low-density energy tails, while the peak longitudinal brightness is preserved. Thus, similar beams have previously been used to lase at the LCLS [26].

\section{CONCLUSIONS}

In this article, we have explored the versatility of laser heater shaping as a technique to manipulate the beam current. Our findings demonstrate that a time dependent laser heater profile is capable of shaping the bunch current to best suit a variety of operational modes. Thanks to the flexibility in tailoring the repetition rate and macropulse structure, we foresee the ability interleave multiple operational modes for multiple beamlines fed by the same linac at high-repetition rate. For example, in a high-repetition rate XFEL operating multiple undulators simultaneously, one could apply different shaping modes to the bunches going to each beamline. 
We have used simple analysis followed by start-to-end simulations to give a broad understanding of the capabilities of this technology. The simple kinetic analysis we present is of interest for giving physical intuition to the process by which laser heater shaping acts on the beam. It shows that, for small modulations, the modulation amplitude scales linearly with the laser power and has a shape given by the second derivative of the power profile. For larger modulations, the current valleys begin to saturate and the peaks steepen. The simplified model we use is helpful for optimizing simulations and will be instructive when building online controls to help integrate laser heater shaping into an operational context.

Our start-to-end simulations illustrate how laser-heater shaping could be used to optimize the beam at LCLS-II. Each case study we discuss optimizes the beam for a unique purpose: to create current spikes for attosecond lasing, to make a bunch train, and finally to suppress caustics and reduce CSR induced emittance growth. Furthermore, we have shown that these techniques can work across a variety of accelerator beamlines at the LCLS-II facility, indicating the robustness of the technique. Our results provide a basis for understanding the wide scope of laser-heater shaping, and thus pave the way for XFEL facilities to simultaneously optimize performance for distribution to multiple users.

\section{ACKNOWLEDGMENTS}

The authors would like to thank Zhirong Huang for useful discussions and suggestions. This work was supported by the Basic Energy Sciences Accelerator and Detector Research program under Contract No. DEAC02-76SF00515, and the Office of Basic Energy Science Accelerator and Detector Research Program.

[1] E. Allaria et al., Highly coherent and stable pulses from the FERMI seeded free-electron laser in the extreme ultraviolet, Nat. Photonics 6, 699 (2012).

[2] W. Decking et al., A MHz-repetition-rate hard X-ray freeelectron laser driven by a superconducting linear accelerator, Nat. Photonics 14, 391 (2020).

[3] P. Emma et al., First lasing and operation of an ångstromwavelength free-electron laser, Nat. Photonics 4, 641 (2010).

[4] L. Giannessi et al., Self-amplified spontaneous emission for a single pass free-electron laser, Phys. Rev. ST Accel. Beams 14, 060712 (2011).

[5] T. Ishikawa et al., A compact X-ray free-electron laser emitting in the sub-ångström region, Nat. Photonics 6, 540 (2012).

[6] H.-S. Kang et al., Hard X-ray free-electron laser with femtosecond-scale timing jitter, Nat. Photonics 11, 708 (2017).

[7] E. Prat et al., A compact and cost-effective hard X-ray freeelectron laser driven by a high-brightness and low-energy electron beam, Nat. Photonics 14, 748 (2020).
[8] Y. Ding, Beam shaping to improve the free-electron laser performance at the Linac Coherent Light Source, Phys. Rev. Accel. Beams 19, 100703 (2016).

[9] E. Roussel, E. Ferrari, E. Allaria, G. Penco, S. Di Mitri, M. Veronese, M. Danailov, D. Gauthier, and L. Giannessi, Multicolor High-Gain Free-Electron Laser Driven by Seeded Microbunching Instability, Phys. Rev. Lett. 115, 214801 (2015).

[10] J. Duris et al., Tunable isolated attosecond X-ray pulses with gigawatt peak power from a free-electron laser, Nat. Photonics 14, 30 (2020).

[11] S. Huang, Y. Ding, Y. Feng, E. Hemsing, Z. Huang, J. Krzywinski, A. Lutman, A. Marinelli, T. Maxwell, and D. Zhu, Generating Single-Spike Hard X-ray Pulses with Nonlinear Bunch Compression in Free-Electron Lasers, Phys. Rev. Lett. 119, 154801, 2017.

[12] A. Malyzhenkov, Y. P. Arbelo, P. Craievich, P. Dijkstal, E. Ferrari, S. Reiche, T. Schietinger, P. Juranić, and E. Prat, Single-and two-color attosecond hard x-ray free-electron laser pulses with nonlinear compression, Phys. Rev. Research 2, 042018 (2020).

[13] Z. Zhang, J. Duris, J. P. MacArthur, A. Zholents, Z. Huang, and A. Marinelli, Experimental demonstration of enhanced self-amplified spontaneous emission by photocathode temporal shaping and self-compression in a magnetic wiggler, New J. Phys. 22, 083030 (2020).

[14] G. Andonian, S. Barber, F. O'Shea, M. Fedurin, K. Kusche, C. Swinson, and J. Rosenzweig, Generation of Ramped Current Profiles in Relativistic Electron Beams Using Wakefields in Dielectric Structures, Phys. Rev. Lett. 118, 054802 (2017).

[15] G. Loisch et al., Observation of High Transformer Ratio Plasma Wakefield Acceleration, Phys. Rev. Lett. 121, 064801 (2018).

[16] P. Emma, K. Bane, M. Cornacchia, Z. Huang, H. Schlarb, G. Stupakov, and D. Walz, Femtosecond and Subfemtosecond X-Ray Pulses from a Self-Amplified SpontaneousEmission-Based Free-Electron Laser, Phys. Rev. Lett. 92, 074801 (2004).

[17] A. Marinelli, J. MacArthur, P. Emma, M. Guetg, C. Field, D. Kharakh, A. A. Lutman, Y. Ding, and Z. Huang, Experimental demonstration of a single-spike hard-X-ray freeelectron laser starting from noise, Appl. Phys. Lett. 111, 151101 (2017).

[18] P. Emma, J. Frisch, Z. Huang, A. Marinelli, T. Maxwell, H. Loos, Y. Nosochkov, T. Raubenheimer, J. Welch, and L. Wang, Linear accelerator design for the LCLS-II FEL facility, in Proceedings of the 36th International FreeElectron Laser Conference, Basel, 2014 (JACoW, Geneva, 2014).

[19] Z. Huang, M. Borland, P. Emma, J. Wu, C. Limborg, G. Stupakov, and J. Welch, Suppression of microbunching instability in the linac coherent light source, Phys. Rev. ST Accel. Beams 7, 074401 (2004).

[20] E. L. Saldin, E. A. Schneidmiller, and M. V. Yurkov, Longitudinal space charge-driven microbunching instability in the TESLA Test Facility linac, Nucl. Instrum. Methods Phys. Res., Sect. A 528, 355 (2004).

[21] M. Hamberg, D. Dancila, M. Jacewicz, M. Karlsson, M. Kuittinen, J. Ögren, A. Rydberg, E. Vargas Catalan, and I. Vartiainen, Dielectric laser acceleration setup design, 
grating manufacturing and investigations into laser induced RF cavity breakdowns (JACOW, Geneva, Switzerland, 2018), pp. 365-367, ISBN 978-3-95450-179-3.

[22] Z. Huang et al., Measurements of the linac coherent light source laser heater and its impact on the x-ray free-electron laser performance, Phys. Rev. ST Accel. Beams 13, 020703 (2010).

[23] J. Lee, J.-H. Han, S. Lee, J. Hong, C. H. Kim, C. K. Min, and I. S. Ko, PAL-XFEL laser heater commissioning, Nucl. Instrum. Methods Phys. Res., Sect. A 843, 39 (2017).

[24] M. Pedrozzi, M. Calvi, R. Ischebeck, S. Reiche, C. Vicario, B. D. Fell, and N. Thompson, The laser heater system of SwissFEL, in Proceedings of FEL'14, Basel, Switzerland, 2014 (JACoW, Geneva, 2014), pp. 871-877.

[25] S. Spampinati et al., Laser heater commissioning at an externally seeded free-electron laser, Phys. Rev. ST Accel. Beams 17, 120705 (2014).

[26] A. Marinelli et al., Optical Shaping of X-ray Free-Electron Lasers, Phys. Rev. Lett. 116, 254801 (2016).

[27] A. D. Brynes et al., Microbunching instability characterisation via temporally modulated laser pulses, arXiv: 2010.00252.

[28] Z. Zhang, L. Yan, Y. Du, W. Huang, C. Tang, and Z. Huang, Generation of high-power, tunable terahertz radiation from laser interaction with a relativistic electron beam, Phys. Rev. Accel. Beams 20, 050701 (2017).

[29] J. Lumeau, L. Glebov, and V. Smirnov, Tunable narrowband filter based on a combination of Fabry-Perot etalon and volume Bragg grating, Opt. Lett. 31, 2417 (2006).

[30] L. J. Hornbeck, Spatial Light Modulator and Method (Google Patents, 1991).

[31] P. Tournois, Acousto-optic programmable dispersive filter for adaptive compensation of group delay time dispersion in laser systems, Opt. Commun. 140, 245 (1997).

[32] S. Carbajo and K. Bauchert, Power handling for LCoS spatial light modulators, in Laser Resonators, Microresonators, and Beam Control XX (International Society for Optics and Photonics, 2018), Vol. 10518, p. 105181R.

[33] R. Lemons, W. Liu, J. C. Frisch, A. Fry, J. Robinson, S. Smith, and S. Carbajo, Integrated structured light architectures, arXiv:2003.14400.

[34] D. Lin, J. Carpenter, Y. Feng, S. Jain, Y. Jung, Y. Feng, M. N. Zervas, and D. J. Richardson, Reconfigurable structured light generation in a multicore fibre amplifier, Nat. Commun. 11, 3986 (2020).

[35] E. Hemsing, G. Stupakov, D. Xiang, and A. Zholents, Beam by design: Laser manipulation of electrons in modern accelerators, Rev. Mod. Phys. 86, 897 (2014).

[36] N. Liebster, J. Tang, D. Ratner, W. Liu, S. Vetter, Z. Huang, and S. Carbajo, Laguerre-Gaussian and beamlet array as second generation laser heater profiles, Phys. Rev. Accel. Beams 21, 090701 (2018).

[37] J. Tang et al., Laguerre-Gaussian Mode Laser Heater for Microbunching Instability Suppression in Free-Electron Lasers, Phys. Rev. Lett. 124, 134801 (2020).

[38] M. Borland, ELEGANT: A flexible SDDS-compliant code for accelerator simulation, Technical Report No. LS-287 (Argonne National Lab., IL (US), 2000).
[39] T. Driver, Attosecond transient absorption spooktroscopy: A ghost imaging approach to ultrafast absorption spectroscopy, Phys. Chem. Chem. Phys. 22, 2704 (2020).

[40] D. Ratner, J. Cryan, T. Lane, S. Li, and G. Stupakov, PumpProbe Ghost Imaging with SASE FELs, Phys. Rev. X 9, 011045 (2019).

[41] C. Evain, C. Szwaj, S. Bielawski, M. Hosaka, Y. Takashima, M. Shimada, S. Kimura, M. Katoh, A. Mochihashi, T. Takahashi, and T. Hara, Laser-induced narrowband coherent synchrotron radiation: Efficiency versus frequency and laser power, Phys. Rev. ST Accel. Beams 13, 090703 (2010).

[42] A. S. Weling and D. H. Auston, Novel sources and detectors for coherent tunable narrow-band terahertz radiation in free space, J. Opt. Soc. Am. B 13, 2783 (1996).

[43] D. Xiang, Y. Ding, T. Raubenheimer, and J. Wu, Modelocked multichromatic $\mathrm{x}$ rays in a seeded free-electron laser for single-shot x-ray spectroscopy, Phys. Rev. ST Accel. Beams 15, 050707 (2012).

[44] M. Venturini, Models of longitudinal space-charge impedance for microbunching instability, Phys. Rev. ST Accel. Beams 11, 034401 (2008).

[45] There is some freedom in how to define $\sigma_{z}$ and thus choose which part impedance to neglect. For a Gaussian we have the simple identification with the rms longitudinal beam size, in which case the approximation is accurate provided $\frac{\gamma \sigma_{z}}{r_{b}} \gg 1$. For other distributions one must choose a reasonable cutoff-frequency, but since $\sigma_{z}$ appears only in the logarithm, the result is not sensitive to the exact choice.

[46] M. Woodley, LCLS lattice description, https://www.slac .stanford.edu/grp/ad/model/lcls.html.

[47] J. Qiang, Y. Ding, P. Emma, Z. Huang, D. Ratner, T. Raubenheimer, M. Venturini, and F. Zhou, Start-to-end simulation of the shot-noise driven microbunching instability experiment at the Linac Coherent Light Source, Phys. Rev. Accel. Beams 20, 054402 (2017).

[48] L. Wang, P. Emma, J. Qiang, and T. Raubenheimer, Benchmark of ELEGANT and IMPACT, in Proceedings of the 37th International Free Electron Laser Conference, 2015 (JACoW, Geneva, 2015).

[49] N. Sudar, Y. Ding, Y. Nosochkov, K. Bane, and Z. Zhang, Octupole based current horn suppresion in multi-stage bunch compression with emittance growth correction, arXiv:2009 .02020 .

[50] T. Charles, D. Paganin, and R. Dowd, Caustic-based approach to understanding bunching dynamics and current spike formation in particle bunches, Phys. Rev. Accel. Beams 19, 104402 (2016).

[51] K. L. F. Bane, F.-J. Decker, Y. Ding, D. Dowell, P. Emma, J. Frisch, Z. Huang, R. Iverson, C. Limborg-Deprey, H. Loos, H.-D. Nuhn, D. Ratner, G. Stupakov, J. Turner, J. Welch, and $\mathrm{J}$. Wu, Measurements and modeling of coherent synchrotron radiation and its impact on the Linac Coherent Light Source electron beam, Phys. Rev. ST Accel. Beams 12, 030704 (2009).

[52] J. P. MacArthur, J. Duris, Z. Zhang, A. Lutman, A. Zholents, X. Xu, Z. Huang, and A. Marinelli, Phase-Stable Self-Modulation of an Electron Beam in a Magnetic Wiggler, Phys. Rev. Lett. 123, 214801 (2019). 